\title{
Tightening the Biological Constraints on Gradient-Based Predictive Coding
}

\author{
Nick Alonso* \\ Emre Neftci* \\ nalonso2@uci.edu \\ eneftci@uci.edu \\ University of California, Irvine \\ Irvine, CA, USA
}

\begin{abstract}
Predictive coding (PC) is a general theory of cortical function. The local, gradient-based learning rules found in one kind of PC model have been shown to closely approximate back-propagation under certain conditions [18, 26, 32]. This finding suggests that this PC model may be useful for understanding how the brain solves the credit assignment problem. The model may also be useful for developing local learning algorithms that are compatible with neuromorphic hardware. In this paper, we modify this PC model so that it better fits biological constraints, including the constraints that neurons can only have positive firing rates and the constraint that synapses only flow in one direction. We also compute the gradientbased weight and activity updates given the modified activity values. We show that, under certain conditions, these modified PC networks perform as well or nearly as well on MNIST data as the unmodified PC model and networks trained with back-propagation.
\end{abstract}

\section{CCS CONCEPTS}

- Computing methodologies $\rightarrow$ Neural networks.

\section{KEYWORDS}

Predictive Coding, Gradient-based Learning, Local Learning, Synaptic Plasticity

\section{ACM Reference Format:}

Nick Alonso and Emre Neftci. 2021. Tightening the Biological Constraints on Gradient-Based Predictive Coding. In Oakridge, IL '21: International Conference on Neuromorphic Systems, Fuly 27-29, 2021, Oakridge, IL. ACM, New York, NY, USA, 9 pages. https://doi.org/10.1145/3477145.3477148

\section{INTRODUCTION}

Predictive coding (PC) is a general theory of the function of topdown and bottom-up processing in the neocortex [10, 24, 28]. According to the theory, a central function of top-down projections, which are connections leading from higher to lower level areas of the cortical hierarchy, is to predict neural activity. Differences

*Both authors contributed equally to this research.

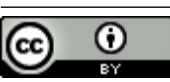

This work is licensed under a Creative Commons Attribution International 4.0 License.

Oakridge '21, fuly 27-29, 2021, Oakridge, IL

(c) 2021 Copyright held by the owner/author(s).

ACM ISBN 978-1-4503-8691-3/21/07.

https://doi.org/10.1145/3477145.3477148 between the predictions and the actual activity are computed and encoded in error neurons, which feed the errors back up the cortical hierarchy from lower to higher level areas. Learning dynamics and neural activity updates seek to minimize the prediction errors. While more empirical testing of PC theory is needed to validate it, the theory has seen significant empirical support and is consistent with much of what we know about neuroanatomy [12, 14, 31], making it a potential basis for a general theory of how the neocortex learns and performs inference.

One PC model, based on Rao and Ballard's seminal neural model [25], updates activities using the gradients of the squared prediction errors at each level. This model has been shown to have interesting formal connections to probabilistic inference and the backpropagation of error (BP) algorithm.

For example, the weight and activity updates for gradient-based PC networks have been shown to optimize an objective function known as variational free energy or, equivalently, the evidence lower bound $[3,9,10]$. Models that seek to minimize free energy perform a kind of approximate Bayesian inference known as variational inference, which estimates the Bayesian posterior through optimization methods [2]. Under this probabilistic interpretation, top-down connections in PC networks learn an approximate, hierarchical generative model. Bottom-up and lateral error signals iteratively optimize an estimate of the posterior.

Connections between the PC and the BP algorithm were first discussed by Whittington and Bogacz [32] who showed that gradientbased PC networks, which were originally developed for unsupervised learning tasks, could be adapted for supervised tasks, and that weight updates performed at convergence on these networks approximate the weight updates performed by BP. Other formal connections between PC and BP have recently been made as well, which show that under certain constraints, PC networks can be made to approximate BP very closely or exactly (e.g. [18, 26]). In all of these cases, PC networks performed as well as networks trained with BP on supervised tasks.

These results are interesting given that $\mathrm{PC}$ networks, unlike $\mathrm{BP}$, use Hebbian-like local learning rules, making PC networks a potential biologically plausible alternative to BP. Instead of using a separate backward pathway, like BP, to propagate global error gradients, PC networks propagate error gradients along the same forward/topdown pathway that produce the errors (see figure 1). Neuron populations accumulate information about the back-propagated global error gradients. As these neurons accumulate global error information, local prediction errors begin to form (which point roughly along the global error gradients) and are encoded in local error 
neurons. Weights are then updated with a local learning rule that involves postsynaptic error neuron activity and presynaptic activity.

In addition to providing a general theory of learning and inference in the brain, we suspect this PC model could advance neuromorphic computing by providing a path toward local learning algorithms that are compatible with neuromorphic hardware $[5,8,23]$. For example, one possibility would involve integrating surrogate gradient methods, used to train spiking neural networks [21], with gradient-based PC models to create spiking PC networks that learn using local learning rules.

Despite its ability to explain various neuro-physiological data, gradient-based PC models have certain biological implausibilities. The model, for example, (as we explain below) is a rate coding network that allows neuron activities to have positive and negative values. Of course, biological neurons cannot have negative spiking rates. Additionally, the model uses the transposes of the forward/top-down weights to propagate information backward, which seems to imply bidirectional synapses. This raises the question of whether these biological implausibilities can be removed from the gradient-based PC model without affecting performance or its formal connection to $\mathrm{BP}$ and probabilistic inference. If gradientbased PC is unable to fit biological constraints without significantly affecting performance or its formal connections to $\mathrm{BP}$ and Bayesian inference, it would affect its plausibility as a neural model, as a theory of how the brain solves the credit assignment problem, and as an implementation of probabilistic interpretations of cortical function. It may also suggest that approximating this model in spiking neurons may not lead to favorable results.

In this paper, we develop methods for removing these biological implausibilities from gradient-based PC models without negatively affecting performance or removing any formal connections the network has to BP. We test these modifications on a supervised learning task using the MNIST dataset and show that these modified versions of PC model work as well as the original PC model and BP.

\section{GRADIENT-BASED PREDICTIVE CODING}

In this section, we describe a gradient-based PC network based on Rao and Ballard's seminal neural model [25]. In general, PC models involve top-down and bottom-up pathways. Top-down pathways, which in supervised PC networks are analogous to forward paths in feedforward networks (see figure 1, propagate predictions of neural activity to levels below. Bottom-up pathways propagate prediction errors. Rao and Ballard's model uses the propagated errors to perform gradient descent over the activities, which minimizes the squared prediction errors at each level.

\subsection{The Gradient-Based PC Model}

\begin{tabular}{||cc||}
\hline Term & Value \\
\hline \hline$a_{l}$ & activity at level $l$ \\
\hline$e_{l}$ & prediction error at level $l$ \\
\hline$p_{l}$ & prediction at level $l$ \\
\hline$W_{l}$ & synaptic weights from $l$ to $l+1$ \\
\hline
\end{tabular}

There are two kinds of neurons in the PC model: activity neurons and prediction error neurons. Activity neurons represent features of the input, while error neurons encode differences between predictions of activity neurons and the actual values of activity neurons. Predictions of activities at level $l$ are passed from level $l-1$ according to the following equation:

$$
p_{l}=W_{l-1} a_{l-1} .
$$

A local prediction error is then computed and encoded in error neurons. Error neurons are shown as the red nodes in figure 1. The error is the difference between the activity and the prediction, which is first passed through an element-wise non-linearity $f$.

$$
e_{l}=a_{l}-f\left(p_{l}\right)
$$

The weight matrices $W_{l}$ are trained using this local error according to:

$$
W_{l} \leftarrow \alpha \Delta W_{l}+W_{l}, \text { where } \Delta W_{l}=e_{l+1} f^{\prime}\left(p_{l+1}\right) a_{l}^{T} .
$$

$\Delta W_{l}$ is multiplied by a small learning rate $\alpha$ before being added to the weights, where $0<\alpha<1$. This update is equivalent to taking the gradient of the squared prediction error $\frac{1}{2} e_{l}^{2}$ with respect to the weights that produced the prediction [25]. This update is local and Hebbian-like because it depends mainly on presynaptic activity neurons and post-synaptic error neurons and post-synaptic information about the prediction.

PC networks update their activities to minimize the the squared prediction errors at each level using gradient descent, which can be seen as minimizing variational free energy, under certain assumptions [10, 11]. At each time-step, PC networks update their activities according to:

$$
a_{l} \leftarrow \beta \Delta a_{l}+a_{l} \text {, where } \Delta a_{l}=\underbrace{W_{l}^{T} e_{l+1} f^{\prime}\left(p_{l+1}\right)}_{\text {Bottom-Up Error }}-\underbrace{e_{l}}_{\text {Top-Down Error }} .
$$

Here, $f^{\prime}$ is the gradient of the non-linearity. Updating activities at level $l$ is equivalent to performing gradient ascent over the activities using the gradient of the squared prediction error at level $l$ and the next level below $l+1$. We call the error gradient from the level below the bottom-up error, as errors are typically described as moving up levels in a bottom up fashion. We call the error gradient from the same level $l$ the top-down error, as it is the error produced by the top-down prediction. Because one is performing gradient descent, the computed gradient must be multiplied by a small value $\beta$, where $0<\beta<1$, before adding to the activities. We call $\beta$ the inference rate because, unlike learning rates, $\beta$ is not used for updating weighted connections (i.e. for learning). Rather, it determines how quickly the network performs iterative inference over activities. One typically wants the activities to update quicker than the weights, so $\beta$ should be larger than $\alpha$. This is because one wants activities to converge quickly to an optimal representation of the input, while learning (i.e. weight change) should occur slowly to ensure generalization ability.

The algorithm used to train PC networks for supervised learning tasks is described in detail below (see algorithm 1). In sum, the activities at the first/top layer are clamped to input data (e.g. images), while activities at the bottom layer are clamped to global target. The network is then allowed to propagate signals forward and 
Back-Propagation

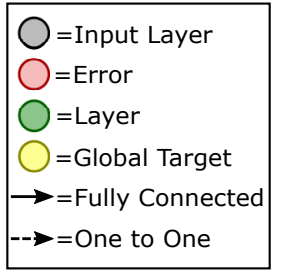

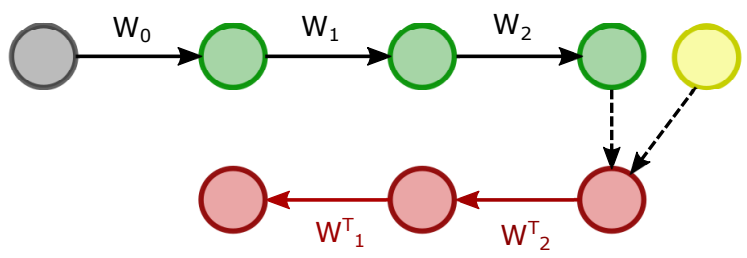

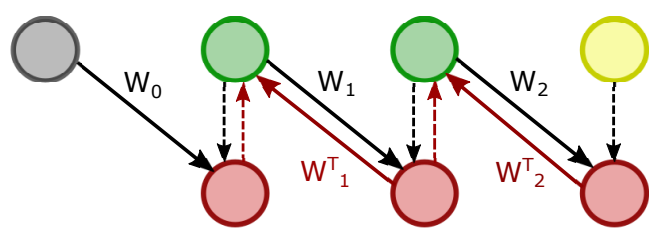

Figure 1: A comparison of a feed-forward network trained with BP (left) and a PC network trained with local learning rules (right). The feedforward network propagates signals forward one layer at a time (gray and green nodes). Its output layer explicitly encodes its prediction of the global target. This prediction is compared to the global target to produce a global error, whose gradient is back-propagated to each hidden layer along a separate backward pathway (red nodes and arrows). PC networks, on the other hand, use two separate kinds of neurons: activity neurons (gray, green, and yellow nodes) and error neurons (red nodes). Activity neurons produce predictions of the activities at the level below. These predictions are fed directly into error neurons, which compute the prediction error. Prediction error gradients are then fed backward and laterally to update the activity neurons, which accumulate error gradients over time. The activity neurons at the bottom level are clamped to the global target. Global prediction errors are computed at this level and fed backward and added to the previous layer's activity neurons, which creates local prediction errors, which are fed backward again, and so on. This process allows global error information to travel backward through the PC network.

back until convergence, at which point it is trained. Global error information propagates from the bottom level to previous layers during this process and becomes encoded in local error neurons, which leads weight updates to incorporate global error information. For more details see figure 1. See also Whittington and Bogacz [32].

\section{PREDICTIVE CODING CONSTRAINED}

We now describe our modified PC network. Each modification to the network is made to make the model fit better with neurobiology. However, we wish to make these changes without losing the ability of the network to propagate gradients and perform similarly to BP.

\subsection{Predictive Coding without Weight Transport}

Equation 6 shows that error gradients propagated back through the network require using the transpose of the forward weights. Using the transpose of forward weights to propagate errors is generally considered biologically implausible as it implies a bidirectional synapse. Transposing a weight matrix is also problematic from a neuromorphic hardware implementation perspective because it requires the duplication and synchronization of the weights on the pre-synaptic and the post-synaptic side.

The weight transport problem has been tackled in the context of BP for conventional deep networks using approximations such as random feedback weights [17] and local loss functions [1, 16, 20]. These approximations underperform slightly compared to exact gradient back-propagation, but do not require a symmetric transpose of the network weights.

We propose two modifications to PC networks that avoid this problem, which build off of previous work done on the weight transport problem for BP.

In the first network model, which we call Rand-PC, we replace the $W^{T}$ in equation 6 with a random matrix $B$ of the same size. Replacing weight transposes with random feedback matrices has been shown to work reasonably well for approximating backpropagation in conventional deep networks [17,33]. In the results section, we show it works nearly as well as BP in our predictive coding networks.

In the second network model, which we call the Kollen-Pollack PC (KP-PC), we use a method developed by Kolen and Pollack [15], and extended by Akrout et al. [1], to train backward matrices so that they converge to the transposes of the forward matrices. Like Rand-PC, with KP-PC we replace the weight transpose in equation 6 with a separate weight matrix $B_{l}$. This weight matrix is then trained with the transpose of the update to the forward $W_{l}$. Kollen and Pollack build on the simple idea that, if one makes the same weight update to two matrices, $W$ and $B$, the two matrices will grow more similar. However, due to numerical precision errors, the two matrices in practice eventually diverge. To solve this problem in conventional ANN, Kolen and Pollack add a small decay term to both updates. They show that $W$ and $B$ will eventually converge to (nearly) the same matrix.

$$
\Delta W_{l}=A_{l}-\gamma W_{l}, \Delta B_{l}=A_{l}^{T}-\gamma B_{l}
$$

where $0<\gamma<1$ is a small decay rate and $A_{l}$ is the weight adjustment. Acrout et al. [1] point out that these weight updates are local for the forward and backward matrices, if each weight matrix is connected to the same two populations of neurons. Interestingly, as can be seen from the equations in section 2 and figure $1, \mathrm{PC}$ networks require just this structure, where forward weights take input from activities at level $l$ and output to error neurons at level $l+1$, while backward weights to the opposite.

Previous work has incorporated separate, trained feedback weights into PC networks before. In particular, Millidge et al. [19] and Ororbia [22] trained separate feedback weights with simple Hebbian learning rules. However, simple Hebbian updates do not guarantee the feedforward and backward matrices will become symmetric. We present the KP rule here to show an alternative local learning 
rule can be used to train feedback weights in PC networks that does have this guarantee.

\subsection{Biologically Constrained Activity Neurons}

The values of neurons in the gradient-based PC model are continuous valued. Typically, these values are interpreted as firing rates of the neurons. PC networks, however, have activity neurons that allow for negative activity values. This is in large part because updates to activities are proportional to the local error gradients w.r.t. those activities, and gradient steps are linear. This means that overtime, even if activity neurons are initialized to positive values, the activity neurons may change to have negative values.

In biological neurons and artificial spiking neurons, of course, firing rates are never negative. Thus, from a neural modelling perspective it is desirable to have neurons with non-negative activities. This raises the question of how well PC models work under the constraint that activities are non-negative. To test this, we enforce positive activation values by clamping the activities after each update, such that all negative values become zero. The activity update now becomes

$$
a_{l} \leftarrow \min \left(0, \beta \Delta a_{l}+a_{l}\right)
$$

Clamping activities in this way will lead to a loss of gradient information whenever there exist negative activity values. Activity neurons are accumulator neurons that accumulate error gradients over time. If some of the accumulated gradients push activity values negative, clamping these negative values to zero will erase the gradient information the pushed activities negative and thus will affect how the network learns.

To help prevent a loss of gradient information due to the clamping function, we simply add a constant, positive bias term to the model. In equation 2, for example, the error $e_{l}$ is now equal to $a_{l}-f\left(p_{l}\right)+b$. For the error encoding schemes outlined in the next section, one simply replaces $f\left(p_{l}\right)$ with $f\left(p_{l}\right)+b$ wherever $f\left(p_{l}\right)$ is present. No other changes to the activity updates or learning rules are needed. The bias works by pushing the predictions to be more positive. If $b$ is large enough, $f\left(p_{l}\right)+b$ will always be positive. This bias will, consequently, prevent activities from being initialized to negative values. It will also prevent activities from trending toward negative values as they accumulate error gradients, through the top-down error (second term in equation 2). The top-down error pulls the activities toward the predictions at the same level. If predictions are always non-zero, they will always pull activities toward non-zero values.

\subsection{Biologically Constrained Error Neurons}

Like the activity neurons, error neurons, according to equation 2 , can take on negative values when activity values are over-predicted. For the same reasons listed in the previous section, it is desirable to have error neurons with only positive firing rate values. This raises the question of how prediction errors could be encoded in spiking patterns with non-negative firing rates.

Rao and Ballard [24] and several other scientists (e.g.[13]) hypothesize there could be two kinds of prediction error neurons in the brain. One kind spikes in response to over-predicted values (i.e. negative errors according to equation 2). The other kind spikes in response to under-predicted values (i.e. positive errors according to equation 2). This encoding scheme implies that generally, when prediction errors are large the error neuron activities will be large, and while errors are small error neuron activity is small. These are the sort of error neurons that are assumed to exist in our PC model. Implementing this encoding scheme could, for example, use one set of error neurons that encode only the positive values of $a_{l}-f\left(p_{l}\right)$ (under-predictions) and another set of error neurons encoding only the positive values of $f\left(p_{l}\right)-a_{l}$ (over-predictions). We will call this sort of error encoding scheme subtractive separated encoding since the subtractive error is encoded in two separate kinds of error neurons.

An alternative way to encode errors in spike trains involves setting error neurons to have a baseline activity rate, which is dampened when activities are over-predicted and excited when they are under-predicted. When subtraction is used to dampen and excite error neurons, we call this encoding scheme the subtractive threshold encoding scheme. There may be several ways to implement this in spiking neurons. For example, one could use a constant input of current that causes the error neurons to have a certain resting firing rate (i.e. a firing rate even when errors are absent).

Here we show how a subtractive threshold scheme can be developed in firing rate neurons so weight and activity updates are equivalent to the original Rao and Ballard equations. Consider the following error neuron encoding scheme

$$
e_{l}^{*}=\frac{2}{e_{\max }}\left(\left(a_{l}-f\left(p_{l}\right)-e_{\min }\right)=\frac{2\left(e_{l}-e_{\min }\right)}{e_{\max }} .\right.
$$

Here, $e_{\min }$ is the minimum possible value of $e_{l}$, and $e_{\max }$ is the maximum possible value of $e_{l}-e_{\min }$. There will be a minimum value if activities are clamped to have a minimum value and $f$ is a squashing non-linearity (e.g. sigmoid). For example, if $f$ is a sigmoid and activities are always non-negative, the error $a_{l}-f\left(p_{l}\right)$ will never go below -1 . If there is no maximum activity rate, there is no formal constrain on the maximum error value. However, one can replace $e_{\max }$ with an approximate $e_{\max }$ value to obtain a similar result. For example, we apply the sigmoid function to $p_{l}$, and clip the activities from below. We find activities never go above 1.1, so an approximate $e_{\max }=2.1$. This equation forces all error values to be between 0 and 2, with a baseline firing rate of 1 . One can replace the 2 with another value which determines the maximum firing rate. If one adds a bias $b$ to the predictions, as in the last section, one simply replaces $f(p)$ with $f(p)+b$ in the above equation.

In spiking neurons, the $e_{\min }$ can be thought of as a constant excitatory current source that, absent any other inputs, causes the neurons to spontaneously fire at a constant rate. The $\frac{2}{e_{\max }}$ term can also be seen as a constant input current that performs some form of normalization, which is well known to be a pervasive computation throughout the brain [4].

Weight and activity updates are computed by replacing the $e_{l}$ term in equation 2 and 6 with $\left(\frac{e_{\max }}{2} e_{l}^{*}+e_{\min }\right)$, since $e_{l}=\left(\frac{e_{\max }}{2} e_{l}^{*}+\right.$ $e_{\min }$ ). With these replacements, updates remain local (since they only depend on the local errors, local activities, and constants) and are equivalent to the original equations.

We note that these two error encoding schemes with biological or spiking neurons can be obtained as special cases of population decoding [6], which can be conveniently realized with e.g. the 
neural engineering framework [7]. Our focus on these special cases is due to their efficiency, as they require one or two neurons per encoded error value compared to population of neurons per error value in the general case.

A third way to encode mismatches between predictions and activities is an encoding scheme that involves dividing the activities by the predictions (or vice versa). Spratling [28], for example, developed a firing rate model of predictive coding which uses a division error term that divides activities (element-wise) by the predictions. His model was able to replicate some neurophysiological data, including fine-grained calcium imaging data that seemed to show the existence of neurons in the mouse primary visual cortex that were sensitive to mismatches between actual and predicted visual flow [27]. We call encoding schemes of this form division mismatch encoding.

Spratling's particular model is not formulated to minimize a global loss using its gradients w.r.t. weights and activities. We are interested in working within the framework of gradient ascent as it has proven effective in large neural networks. We thus present an alternative firing rate model with division mismatch encoding that maintains gradient updates on activities and errors. We compute values of the mismatch neurons as follows:

$$
e_{l}^{* *}=\sqrt{\frac{a_{l}+\epsilon}{f\left(p_{l}\right)+\epsilon}} .
$$

The $\epsilon$ is a small constant that prevents division by zero and helps prevent exploding gradients. The term $\frac{a_{l}+\epsilon}{f\left(p_{l}\right)+\epsilon}$ ranges between 0 and $\infty$ (assuming activities and predictions are positive). Overpredictions range between 0 and 1, while under-predictions range between 1 and $\infty$. We find that, although not necessary, adding the square root function improves learning slightly and makes activity updates more stable. When activities equal the predictions, $e_{l}^{* *}$ will equal one, so we develop a cost function that measures the difference between $e_{l}^{* *}$ and 1 (see appendix). Weights and activities are then updated in proportion to the gradient of this new cost function (see appendix). If one adds a bias $b$ to the predictions, as in the last section, one simply replaces $f(p)$ with $f(p)+b$ in the above equation.

\section{RESULTS}

Predictive coding networks can be trained for both supervised and self-supervised learning tasks. In self-supervised learning tasks, the activities of the bottom level activities (level 0 in figure 1) are set to the data (e.g. image), while the activities of the top level (level 4 in figure 1) are set to some constant (e.g. 1). Alternatively, the top level can be removed entirely. The network weights can then be trained online after each update to the activities, or a single weight update can occur after the network activities converge [3].

In what follows, we show the results of training our models a supervised version of PC networks and a training algorithm, which we call inference learning, developed by [32]. In supervised inference learning learning, the bottom level activities of the PC network are clamped to the target, while the top-level activities are clamped to the input data. Activities are initialized by propagating predictions down level by level to level 0 . Activities of hidden layers are set to the predictions at each level: $a_{l}^{\text {initial }}=f\left(p_{l}^{\text {initial }}\right)$. After initialization, activities are optimized. Weight updates can occur

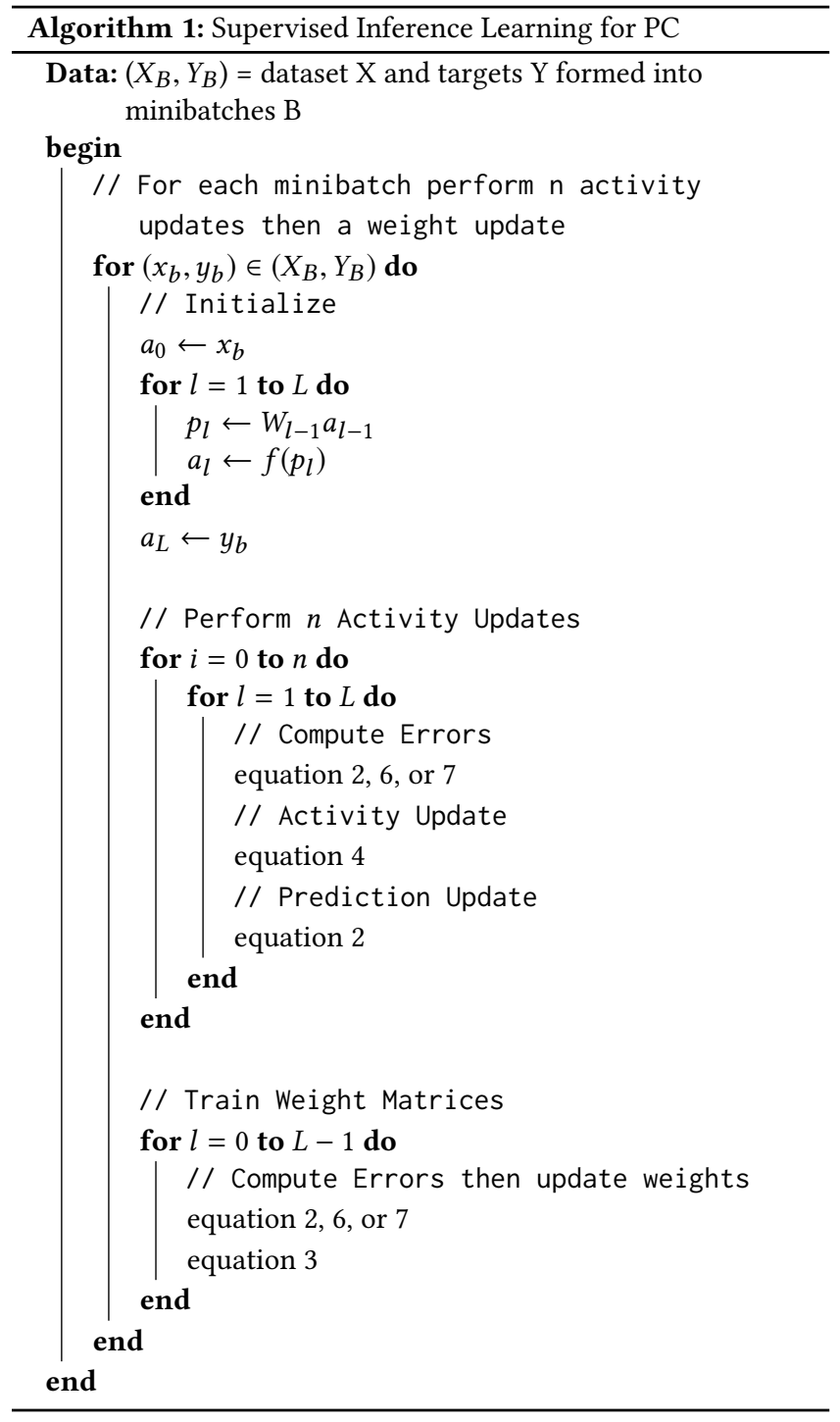

online or after the activities reach convergence (aee algorithm 1). We found activity convergence is slow for MNIST, so instead of waiting for convergence we update weights after 20 activity updates. For fashion-MNIST, the activities converge more quickly, so we update weights after 7 activity updates. When tested, the the activities at level 4 are clamped to the test image, then predictions propagate down to the output layer (level 0 ), where the output is compared to the target.

Inference rates (i.e. $\beta$ in equation 4 ) are set to .1 for MNIST and .025 for Fashion-MNIST. Learning rates are set to .001 . Adam optimizers are used to train all weight matrices. All models use a network with fully connected layers of size of 784-300-300-10. We train the networks to classify images in the MNIST and FashionMNIST data set. Each dataset consists of gray-scale images of size $28 \times 28$. There are 60,000 training images and 10,000 test images in 
each dataset. We show the classification accuracies on the test sets below. ${ }^{1}$

\subsection{PC with Separate Feedback Weights}

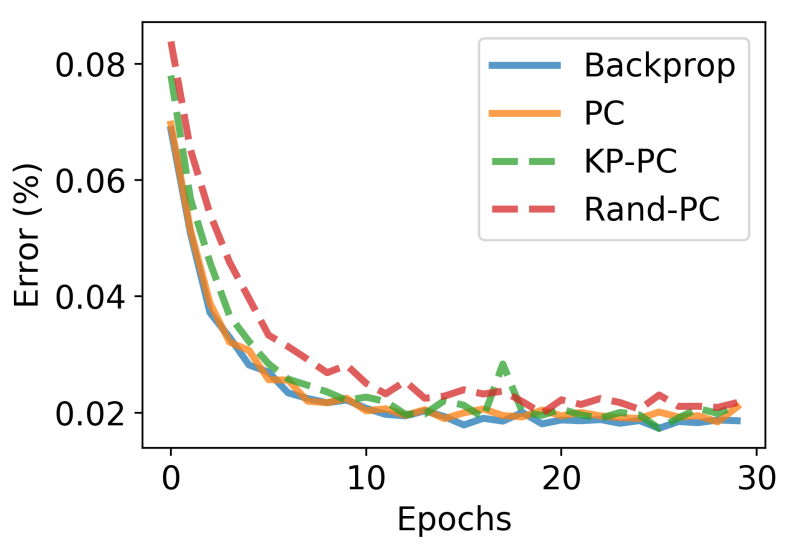

Figure 2: Test errors of PC models with various feedback weights on MNIST with sigmoid activations.

We begin by testing how well PC networks train with the separate feedback matrices discussed in section 2.3. Here all networks use sigmoid activation functions. Rand-PC uses a fixed, random feedback matrix, while the KP-PC uses a weight matrix trained using the rules discussed in section 2.3. The test accuracy, averaged over three runs, is shown after each epoch of training starting after the first training epoch.

We find the PC network, which uses the weight transpose for feedback, performs as well as BP, which replicates the findings of [32]. The KP-PC network also performs as well as backpropagation, while the Rand-PC network only does slightly worse (see table 1). All models achieve a mean accuracy within a standard deviation of $98 \%$. Similar results are found with fashion-MNIST, where these models' mean test errors were within two standard deviations of BP.

\subsection{PC with Constrained Activity Rates}

Next, we test how well PC networks work under the constraint that activity neurons can only take positive firing rate values. As mentioned above, the activity neurons accumulate error gradients from levels below, and this is how error gradients propagate back through the network and are stored over time. Because firing rates are updated linearly, activity values can become negative even when initialized to be positive. It is possible then, that preventing activities from being negative (by clamping them after each update) may erase gradient information stored in the activity neurons, and this may consequently hurt performance. Here we test how severe this potential loss of gradient information is when using different activation functions. All models have sigmoid activations at the

\footnotetext{
${ }^{1}$ Code for models can be found here: https://github.com/nalonso2/Tightening-the-
} Biological-Constraints-on-Gradient-Based-Predictive-Coding output layer, but some models use sigmoid functions at hidden layers while others use Tanh functions at hidden layers.

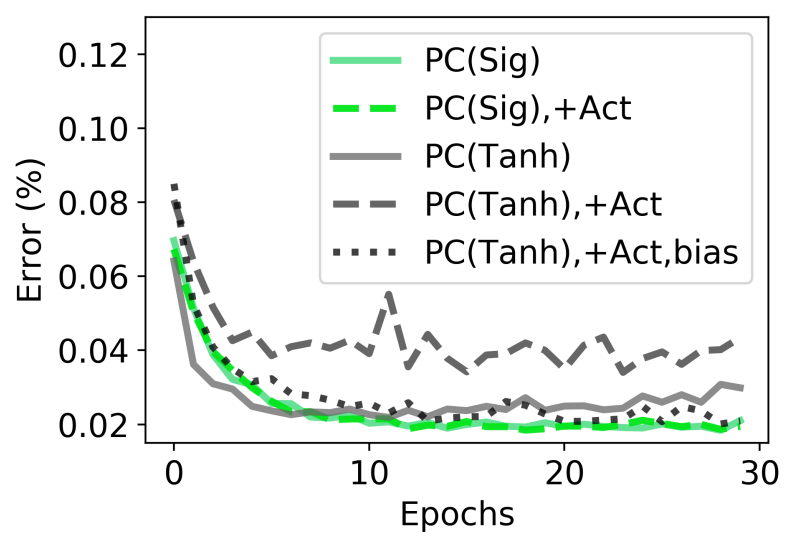

Figure 3: Test errors of PC models on MNIST with activities constrained to be positive (+Act) versus those allowed to go negative. The sigmoid PC network performs well without the bias, because activity values generally stay positive in this network and are thus not affected by clamping. The Tanh PC network, however, allows activities to go negative and thus activities are affected by clamping at training and test time. This negative effect of clamping is removed by adding a positive, constant bias to the predictions.

Figure 3 shows that constraining activities to be positive does not negatively affect the performance of the network when sigmoid activation functions are used. However, there is a small but significant drop in performance when Tanh non-linearities are used. When a small bias is added, however, as specified in section 3.3, the network using Tanh non-linearities sees no drop in performance.

There are a couple reasons why we see this pattern. When the network is initialized, the activities are set to the predictions, and the predictions are first passed through a non-linearity (see 1). At test time, the PC network is initialized, and the predictions at the bottom level are compared against the target. Because sigmoid maps all values to non-negative numbers, the sigmoid PC network will have only positive activities upon initialization. Thus, when activity values are clamped to zeros, no activities are affected because there will be no negative activities. The sigmoid, therefore, prevents the clamping from affecting predictions at test time. Additionally, during training, the sigmoid non-linearity helps prevent the activities from going negative. This is done through the top-down error term (second term in equation 2), which pulls the activities toward the predictions. Since predictions are always positive in the sigmoid PC network, they will always pull activities toward positive values.

When Tanh is applied to predictions, on the other hand, predictions can have negative values. This means the networks can be initialized to negative activity values and the predictions put no pressure on activities to stay positive during inference/training. Thus, during testing, activities may be affected by the clamp, and during training gradient information will be lost when negative 


\begin{tabular}{ccccccc}
\hline Data & Backprop & PC & KP-PC & Rand-PC & PC w/ Div & Rand-PC w/ Div \\
\hline MNIST & $.019\left(1 \times 10^{-3}\right)$ & $.020\left(1 \times 10^{-3}\right)$ & $.019\left(2 \times 10^{-3}\right)$ & $.021\left(1 \times 10^{-3}\right)$ & $.021\left(8 \times 10^{-4}\right)$ & $.022\left(1 \times 10^{-3}\right)$ \\
\hline Fashion-MNIST & $.107\left(2 \times 10^{-3}\right)$ & $.109\left(3 \times 10^{-3}\right)$ & $.116\left(5 \times 10^{-3}\right)$ & $.112\left(3 \times 10^{-3}\right)$ & $.116\left(4 \times 10^{-3}\right)$ & $.116\left(2 \times 10^{-3}\right)$ \\
\hline
\end{tabular}

Table 1: Mean (and standard deviation) of validation errors (\%) for PC models with sigmoid activitations. Three different seeds of each model were trained. Values shown are the means and standard deviations of the validation errors of the last three epochs across training runs.

activity values are set to zero. This negative effect is prevented by adding a constant, positive bias to the predictions that prevent negative activity values.

\subsection{Subtraction versus Division Errors}

In section 4.3, we outlined three error neuron encoding schemes: subtractive separated encoding, subtractive threshold encoding, and division mismatch encoding. We also showed how to compute the gradients for the new encoding schemes. As explained above, the two subtractive error encoding schemes will look quite different in spiking neurons. However, we showed that both of these encoding schemes are mathematically equivalent in the firing rate model, and that the separated subtractive encoding scheme works as well as BP. Here we test how well the division threshold encoding scheme compares to BP.

Positive activity values are required for the division encoding scheme (due to the square-root and logarithm in equations 7 and 8), so we set all models to have positive activity values. We apply sigmoid activation at hidden layers. We still find that adding a small bias is necessary to prevent loss of gradient information, so we add a small bias.

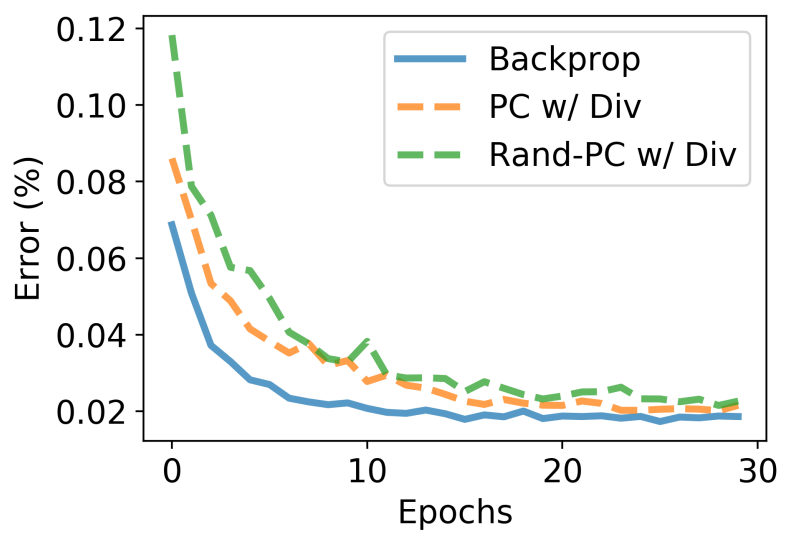

Figure 4: Test errors of PC models on MNIST with division encoding errors.

We can see in figure 4 that the PC models that use division based errors produce comparable results to BP. The division mismatch model that uses true gradients achieves a mean test error of .021 while the division model with random feedback achieves a mean test error of .22 (see table 1), which is only slightly worse than the performance of BP, which had a mean test error of .019. The results are similar for fashion MNIST as well, where both division mismatch models produce mean test errors with less the one percent difference of the mean test error of BP (see 1).

\section{DISCUSSION}

In this paper, we showed that a more biologically constrained version of Rao and Ballard's [25] seminal model of predictive coding performed similarly to $\mathrm{BP}$ on supervised learning tasks using MNIST data. We found this to be true under constraints where 1) separate feedback weights were used to propagate errors, 2) activity values were prevented from going negative, and 3) error neuron activities were prevented from going negative using either division or subtraction based encoding schemes. We also showed how the gradients for the new encoding schemes could be computed and incorporated into the model.

These results suggest that it is likely possible for more biologically constrained models of gradient-based predictive coding to be built using spiking neural networks. We computed the gradients for the division and subtraction threshold error encoding schemes, which prevent error neurons from having negative activity rates. These equations can potentially be used as a basis for forming new equations for spiking neuron models. We also discussed how separated subtraction error encoding could be implemented in spiking neurons. Although spiking models of PC have been previously demonstrated (e.g. [22, 30]), as far as we can find, spiking neural models of PC that utilize gradient-based inference and learning have yet to be developed. Ororbia [22], for example, builds a spiking PC model, which is able to perform some supervised and unsupervised learning tasks. However, this model does not try to approximate gradient propagation, nor does it use spiking error neurons.

Of course, in spiking neurons the true gradients cannot be computed because the step functions used as non-linearities are nondifferentiable. However, surrogate gradient methods [21] used to approximate gradients in spiking neural networks can naturally be incorporated into predictive coding networks. Such surrogategradient spiking PC models could help further develop the empirical hypothesis that predictive coding is the general algorithm the brain uses to solve the credit assignment problem. Additionally, such networks could lead to useful local-learning algorithms that are compatible with neuromorphic hardware. We hope our work here, showing how gradient ascent can be performed in PC networks with only positive firing rates, at least suggests that in theory this can be done.

It is still an open question whether the cortex is performing some form of predictive coding. There is good evidence that top-down 
connections in the cortex do propagate predictions [12, 14, 31] However, the hypothesis that the cortex encodes prediction errors widely in specialized error neurons (which is a key implication of PC) is not yet widely accepted within neuroscience. There is much data that is consistent with the hypothesis that such error neurons exist in the cortex, but none of it is particularly conclusive (for recent review see [31]).

One reason why it has been difficult to locate error neurons is that different error encoding schemes yield different empirical predictions [31]. However, some progress is being made. Spratling [27], for example, recently found that division-based error encoding schemes better fit certain neurophysiological data than the subtractive encoding of the Rao-Ballard model. Spratling did not compare the physiological data to a subtractive threshold encoding scheme, like the one we proposed here (equation 6), so it is unclear whether division error encoding fits with the data better than subtractive schemes generally or only with a particular type of subtractive encoding present in the Rao-Ballard model. Nonetheless, this study shows how we can begin to build evidence in favor of one hypothesis over another.

Our model also illustrates that gradient-based PC is compatible with multiple different kinds of error encoding schemes. So the general hypothesis that some form of gradient-based PC is utilized by the cortex does not depend on there existing a subtractive rather than divisive error encoding. It will, instead, depend on the way the errors are propagated through the cortex and used to affect neural activity and learning. It may be that errors and weights are updated using some other optimization method (e.g. [29]).

More empirical and computational work will be needed to settle these debates. We hope, however, that the work presented here provides new avenues to further develop and test neural models of gradient-based PC and to further develop useful brain-inspired learning algorithms.

\section{CONCLUSION}

$\mathrm{PC}$ is a theory that is of interest to both cognitive science, neuroscience, and engineering. It, for one, is able to explain a wide array of neurophysiological and anatomical data. PC also has potential to provide a path toward understanding how the brain solves the credit assignment problem. We also suggested that the local learning rules used within PC models could lead to useful learning algorithms that are compatible with the constraints neuromorphic hardware. In this paper, we showed that the gradient-based PC model can learn and perform inference well under tighter biological constraints. This further supports the position that PC can be developed in a way that is useful for both neuroscience and neuromorphic computing, and marks a path along which PC can be further developed.

\section{ACKNOWLEDGMENTS}

This work was supported by the National Science Foundation under grant 1652159 and 1823366 (EN).

\section{REFERENCES}

[1] Mohamed Akrout, Collin Wilson, Peter C Humphreys, Timothy Lillicrap, and Douglas Tweed. 2019. Deep learning without weight transport. arXiv preprint arXiv:1904.05391 (2019).
[2] David M Blei, Alp Kucukelbir, and Jon D McAuliffe. 2017. Variational inference: A review for statisticians. Fournal of the American statistical Association 112, 518 (2017), 859-877.

[3] Rafal Bogacz. 2017. A tutorial on the free-energy framework for modelling perception and learning. Journal of mathematical psychology 76 (2017), 198-211.

[4] Matteo Carandini and David J Heeger. 2012. Normalization as a canonical neural computation. Nature Reviews Neuroscience 13, 1 (2012), 51-62.

[5] Mike Davies, Narayan Srinivasa, Tsung-Han Lin, Gautham Chinya, Yongqiang Cao, Sri Harsha Choday, Georgios Dimou, Prasad Joshi, Nabil Imam, Shweta Jain, et al. 2018. Loihi: A neuromorphic manycore processor with on-chip learning. Ieee Micro 38, 1 (2018), 82-99.

[6] Peter Dayan and Laurence F Abbott. 2001. Theoretical neuroscience: computational and mathematical modeling of neural systems. Computational Neuroscience Series.

[7] Chris Eliasmith and Charles H Anderson. 2003. Neural engineering: Computation, representation, and dynamics in neurobiological systems. MIT press.

[8] Simon Friedmann, Johannes Schemmel, Andreas Grübl, Andreas Hartel, Matthias Hock, and Karlheinz Meier. 2016. Demonstrating hybrid learning in a flexible neuromorphic hardware system. IEEE transactions on biomedical circuits and systems 11,1 (2016), 128-142.

[9] Karl Friston. 2010. The free-energy principle: a unified brain theory? Nature reviews neuroscience 11, 2 (2010), 127-138.

[10] Karl Friston and Stefan Kiebel. 2009. Predictive coding under the free-energy principle. Philosophical Transactions of the Royal Society B: Biological Sciences 364, 1521 (2009), 1211-1221.

[11] Karl Friston, James Kilner, and Lee Harrison. 2006. A free energy principle for the brain. Fournal of Physiology-Paris 100, 1-3 (2006), 70-87.

[12] Yanping Huang and Rajesh PN Rao. 2011. Predictive coding. Wiley Interdisciplinary Reviews: Cognitive Science 2, 5 (2011), 580-593.

[13] Georg B Keller and Thomas D Mrsic-Flogel. 2018. Predictive processing: a canonical cortical computation. Neuron 100, 2 (2018), 424-435.

[14] Peter Kok and Floris P de Lange. 2015. Predictive coding in sensory cortex. In An introduction to model-based cognitive neuroscience. Springer, 221-244.

[15] John F Kolen and Jordan B Pollack. 1994. Backpropagation without weight transport. In Proceedings of 1994 IEEE International Conference on Neural Networks (ICNN'94), Vol. 3. IEEE, 1375-1380.

[16] Daniel Kunin, Aran Nayebi, Javier Sagastuy-Brena, Surya Ganguli, Jonathan Bloom, and Daniel Yamins. 2020. Two routes to scalable credit assignment without weight symmetry. In International Conference on Machine Learning. PMLR, 55115521.

[17] Timothy P Lillicrap, Daniel Cownden, Douglas B Tweed, and Colin J Akerman. 2016. Random synaptic feedback weights support error backpropagation for deep learning. Nature communications 7, 1 (2016), 1-10.

[18] Beren Millidge, Alexander Tschantz, and Christopher L Buckley. 2020. Predictive coding approximates backprop along arbitrary computation graphs. arXiv preprint arXiv:2006.04182 (2020).

[19] Beren Millidge, Alexander Tschantz, Anil Seth, and Christopher L Buckley. 2020. Relaxing the constraints on predictive coding models. arXiv preprint arXiv:2010.01047 (2020).

[20] Hesham Mostafa, Vishwajith Ramesh, and Gert Cauwenberghs. 2018. Deep supervised learning using local errors. Frontiers in neuroscience 12 (2018), 608.

[21] Emre O Neftci, Hesham Mostafa, and Friedemann Zenke. 2019. Surrogate gradient learning in spiking neural networks: Bringing the power of gradient-based optimization to spiking neural networks. IEEE Signal Processing Magazine 36, 6 (2019), 51-63.

[22] Alexander Ororbia. 2019. Spiking Neural Predictive Coding for Continual Learning from Data Streams. arXiv preprint arXiv:1908.08655 (2019).

[23] Ning Qiao, Hesham Mostafa, Federico Corradi, Marc Osswald, Fabio Stefanini, Dora Sumislawska, and Giacomo Indiveri. 2015. A reconfigurable on-line learning spiking neuromorphic processor comprising 256 neurons and $128 \mathrm{~K}$ synapses. Frontiers in neuroscience 9 (2015), 141.

[24] Rajesh PN Rao and Dana H Ballard. 1999. Predictive coding in the visual cortex: a functional interpretation of some extra-classical receptive-field effects. Nature neuroscience 2, 1 (1999), 79-87.

[25] Rajesh PN Rao and Dana H Ballard. 1999. Predictive coding in the visual cortex: a functional interpretation of some extra-classical receptive-field effects. Nature neuroscience 2, 1 (1999), 79-87.

[26] Yuhang Song, Thomas Lukasiewicz, Zhenghua Xu, and Rafal Bogacz. 2020. Can the brain do backpropagation? exact implementation of backpropagation in predictive coding networks. Advances in neural information processing systems $33(2020), 22566$.

[27] MW Spratling. 2019. Fitting predictive coding to the neurophysiological data. Brain research 1720 (2019), 146313.

[28] Michael W Spratling. 2008. Predictive coding as a model of biased competition in visual attention. Vision research 48, 12 (2008), 1391-1408.

[29] Michael W Spratling, Kris De Meyer, and R Kompass. 2009. Unsupervised learning of overlapping image components using divisive input modulation. Computational intelligence and neuroscience 2009 (2009). 
[30] Catherine Wacongne, Jean-Pierre Changeux, and Stanislas Dehaene. 2012. A neuronal model of predictive coding accounting for the mismatch negativity. Fournal of Neuroscience 32, 11 (2012), 3665-3678.

[31] Kevin S Walsh, David P McGovern, Andy Clark, and Redmond G O'Connell. 2020 Evaluating the neurophysiological evidence for predictive processing as a model of perception. Annals of the new York Academy of Sciences 1464, 1 (2020), 242.

[32] James CR Whittington and Rafal Bogacz. 2017. An approximation of the error backpropagation algorithm in a predictive coding network with local hebbian synaptic plasticity. Neural computation 29, 5 (2017), 1229-1262.

[33] Will Xiao, Honglin Chen, Qianli Liao, and Tomaso Poggio. 2018. Biologicallyplausible learning algorithms can scale to large datasets. arXiv preprint arXiv:1811.03567 (2018)

\section{A RESEARCH METHODS}

\section{A.1 Division Encoding Cost Function}

The division encoding error $e_{l}^{* *}$ is equal to $\sqrt{\frac{a_{l}}{f\left(p_{l}\right)}}$. Under this encoding scheme, $a_{l}=p_{l}$ when $e_{l}^{* *}=1$. The goal then is to update weights and activities to reduce the difference between $e_{l}^{* *}$ and 1 To do so, we use the following cost function:

$$
C=\sum_{l}^{L} C_{l}, \text { where } C_{l}=\frac{1}{2} \log \left(e_{l}^{* *}\right)^{2} .
$$

When $e_{l}^{* *}=1$ the $\log$ of $e_{l}^{* *}$ will equal 0 so the cost at level $l$ will equal 0 . Deviations from 1 , will lead the cost to increase. We use the $\log$ here, instead of simply subtracting $e_{l}^{* *}$ from 1 because it simplifies the computations of the gradients.

A.1.1 Weight Updates for Division Encoding. Here we derive the gradients of $\mathrm{C}$ at level $l$ with respect to the forward weights at level $l$ :

$$
\begin{aligned}
C_{l} & =\frac{1}{2}\left(\log \left(e_{l}^{* *}\right)\right)^{2}, \\
& =\frac{1}{2}\left(\frac{1}{2}\left(\log \left(a_{l}\right)-\log \left(f\left(p_{l}\right)\right)\right)\right)^{2} .
\end{aligned}
$$

Lets call the term inside the parentheses $u_{l}$, such that $C_{l}=\frac{1}{2} u_{l}^{2}$. Now we need to compute $\frac{\partial C_{l}}{\partial W_{l-1}}$, which can be decomposed using the chain rule as follows.

$$
\frac{\partial C_{l}}{\partial W_{l}}=\frac{\partial C_{l}}{\partial u_{l}} \frac{\partial u_{l}}{\partial f\left(p_{l}\right)} \frac{\partial f\left(p_{l}\right)}{\partial p_{l}} \frac{\partial p_{l}}{\partial W_{l-1}} .
$$

Each term can then be computed individually.

$$
\begin{aligned}
\frac{\partial C_{l}}{\partial u_{l}} & =u_{l}=\frac{1}{2}\left(\log \left(a_{l}\right)-\log \left(f\left(p_{l}\right)\right)\right), \\
\frac{\partial u_{l}}{\partial f\left(p_{1}\right)} & =-1 / f\left(p_{l}\right), \\
\frac{\partial f\left(p_{l}\right)}{\partial p_{l}} & =f^{\prime}\left(p_{l}\right), \\
\frac{\partial p_{l}}{\partial W_{l-1}} & =a_{l-1}^{T} .
\end{aligned}
$$

Now if we combine terms we get the following weight update

$$
\begin{aligned}
\frac{\partial C_{l}}{\partial W_{l}} & =\frac{1}{2}\left(\log \left(a_{l}\right)-\log \left(p_{l}\right)\right)\left(\frac{-1}{f\left(p_{l}\right)}\right) f^{\prime}\left(p_{l}\right) a_{l-1}^{T}, \\
& =-\log \left(\sqrt{\frac{a_{l}}{f\left(p_{l}\right)}}\right) \frac{f^{\prime}\left(p_{l}\right)}{f\left(p_{l}\right)} a_{l-1}^{T}, \\
& =-\log \left(e_{l}^{* *}\right) \frac{f^{\prime}\left(p_{l}\right)}{f\left(p_{l}\right)} a_{l-1}^{T} .
\end{aligned}
$$

We can see this update is the outer product of pre-synaptic and post-synaptic information. In particular, the pre-synaptic activity is multiplied by the error neuron activities, which are first passed through a non-linearity (logarithm) and multiplied by information about the predictions at the same level.

A.1.2 Activity Updates for Division Encoding. Here we derive the gradients of the cost at level $l$ w.r.t. the activities. Like original equation, the gradients w.r.t. $a_{l}$ are derived from the cost at the same level and the next level (i.e. level $l$ and $l+1$ ). The gradient of $C_{l+1}$ w.r.t $a_{l}$ can be decomposed using the chain rule as follows.

$$
\frac{\partial C_{l+1}}{\partial a_{l}}=\frac{\partial C_{l+1}}{\partial u_{l+1}} \frac{\partial u_{l+1}}{\partial f\left(p_{l+1}\right)} \frac{\partial f\left(p_{l+1}\right)}{\partial p_{l+1}} \frac{\partial p_{l+1}}{\partial a_{l}} .
$$

We already computed all of these terms in the last section except for $\frac{\partial p_{l}}{\partial a_{l-1}}$. The gradient $\frac{\partial p_{l}}{\partial a_{l-1}}$ is just equal to $W_{l}^{T}$. This gives us the bottom-up error (the first term in equation 4 ) for the activity update $a_{l}$ :

$$
\frac{\partial C_{l+1}}{\partial a_{l}}=W_{l}^{T} \log \left(e_{l}^{* *}\right) \frac{f^{\prime}\left(p_{l}\right)}{f\left(p_{l}\right)} .
$$

Now we need to compute $\frac{\partial C_{l}}{\partial a_{l}}$. This term can be decomposed using the chain rule as follows.

$$
\frac{\partial C_{l}}{\partial a_{l}}=\frac{\partial C_{l}}{\partial u_{l}} \frac{\partial u_{l}}{\partial a_{l}}
$$

We saw in the last section that $\frac{\partial C_{l}}{\partial u_{l}}$ is equal to $\log \left(e_{l}^{* *}\right) \cdot \frac{\partial u_{l}}{\partial a_{l}}$ is $\frac{1}{a_{l}}$. With these terms we can now compute the top-down error for the activity update (second term in equation 4 ):

$$
\frac{\partial C_{l}}{\partial a_{l}}=\log \left(e_{l}^{* *}\right) \frac{1}{a_{l}}
$$

Combining the top-down and bottom up error now gets us the activity updates under the division encoding schema:

$$
\Delta a_{l}=W_{l}^{T} \log \left(e_{l}^{* *}\right) \frac{f^{\prime}\left(p_{l}\right)}{f\left(p_{l}\right)}-\log \left(e_{l}^{* *}\right) \frac{1}{a_{l}}
$$

